\title{
MODELAGEM E SIMULAÇÃO DE DESTILARIA AUTÔNOMA NO SIMULADOR DE PROCESSOS EMSO
}

\author{
G. C. FONSECA ${ }^{1}$, C. B. B COSTA ${ }^{1}$ e A. J. G. Cruz $^{1}$ \\ ${ }^{1}$ Universidade Federal de São Carlos, Programa de Pós-Graduação em Engenharia Química \\ E-mail para contato: gabrieldcf@gmail.com
}

\begin{abstract}
RESUMO - Implementou-se no simulador de processos EMSO uma destilaria autônoma de etanol hidratado. Foram representadas as etapas de lavagem da cana, extração e tratamento do caldo, preparo do mosto, fermentação e destilação. Duas configurações foram avaliadas para a etapa de fermentação: operação contínua em estado estacionário (quatro reatores em série) e operação batelada alimentada em regime dinâmico (seis reatores em paralelo). Em ambos os casos, os fermentadores foram modelados usando-se a cinética de Ghose e Tyagi e considerando-se uma alimentação de $500 \mathrm{t} / \mathrm{h}$ de cana de açúcar contendo $13 \%$ em massa de sacarose. Após a etapa de tratamento obteve-se uma corrente de $330 \mathrm{t} / \mathrm{h}$ de mosto contendo $18,7 \%$ em massa de sacarose, que resultou em ambos os casos em produções próximas a 30 t/h de etanol 93,5\% em massa.
\end{abstract}

\section{INTRODUÇÃO}

A indústria sucroalcooleira é de grande importância para a economia e para a matriz energética brasileira. Na safra 2012/13 foram produzidas 23,6 bilhões de litros de etanol (CONAB, 2013) e os veículos bicombustíveis correspondiam em março de 2013 a 58\% da frota nacional de veículos leves (UNICA, 2013). No ano de 2011 o etanol combustível (AEHC) e a queima do bagaço da cana foram responsáveis, respectivamente, por $4,7 \%$ e $11,9 \%$ do total de energia consumida no Brasil (EPE, 2012). Junto com a matriz de geração de energia hidrelétrica, a indústria do etanol contribui para estabelecer o Brasil como líder mundial no uso de fontes de energia renováveis e contribui para mitigar o agravamento do efeito estufa. Os biocombustíveis despertam grande interesse também no exterior, tanto que o Brasil perdeu para os Estados Unidos o posto de maior produtor mundial de etanol em 2006 (RFA, 2012). Não por acaso, o processo industrial de produção de etanol é objeto de grande interesse na literatura científica.

Vários trabalhos publicados em anos recentes implementam a simulação de destilarias de etanol de primeira ou segunda geração, como Dias et al. (2012), Furlan et al. (2012), Palacios-Bereche et al. (2013). Todos esses trabalhos, no entanto, modelam o processo de fermentação alcoólica com base em modelos de conversão estequiométrica. O objetivo deste trabalho é implementar a simulação de uma destilaria autônoma de etanol usando um modelo cinético (Ghose e Tyagi, 1979) e duas configurações diferentes: uma para a operação batelada alimentada usando um conjunto de seis fermentadores em paralelo e outra para a operação contínua em regime estacionário usando um conjunto de quatro fermentadores em série. 


\section{METODOLOGIA}

Os modelos dos equipamentos utilizados em uma destilaria autônoma foram desenvolvidos no simulador de processos orientado por equações EMSO (SOARES e SECCHI, 2003). Foram aproveitados os modelos desenvolvidos por Furlan et al. (2012) para representar as unidades de recepção da cana, moagem, tratamento do caldo e destilação. Novos modelos de fermentadores foram criados fazendo uso de equações cinéticas de conversão, além de modelos de acessórios como centrífugas e válvulas com acionamento periódico.

A Figura 1 mostra um diagrama de blocos do processo completo construído no simulador. O bloco referente à fermentação corresponde aos subprocessos detalhados nas Figuras 2 e 3 , que correspondem respectivamente a uma configuração com seis fermentadores em paralelo em operação batelada alimentada e regime dinâmico e a uma configuração com quatro fermentadores em série em operação contínua e regime estacionário.

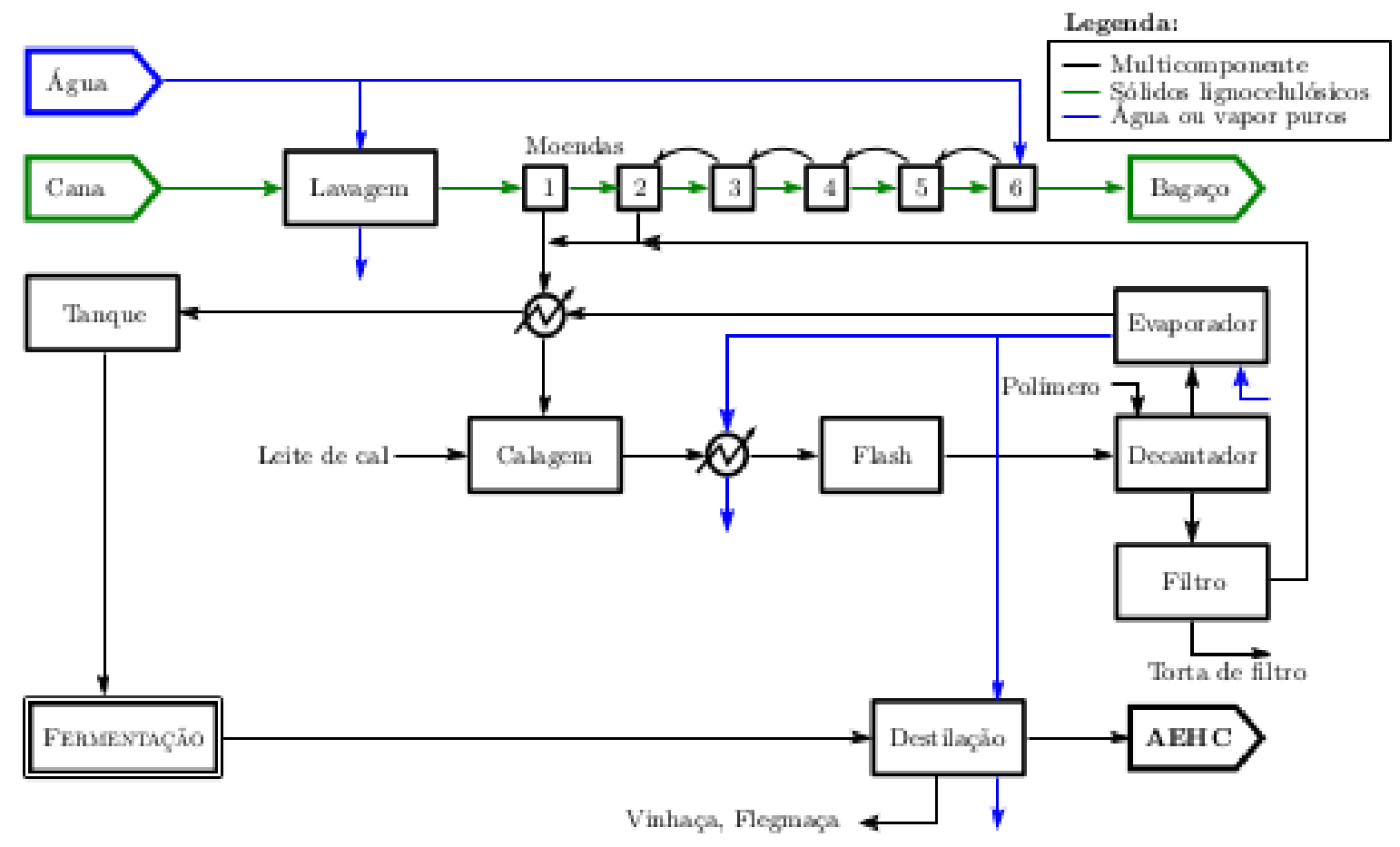

Figura 1 - Diagrama de blocos da destilaria autônoma implementada. 


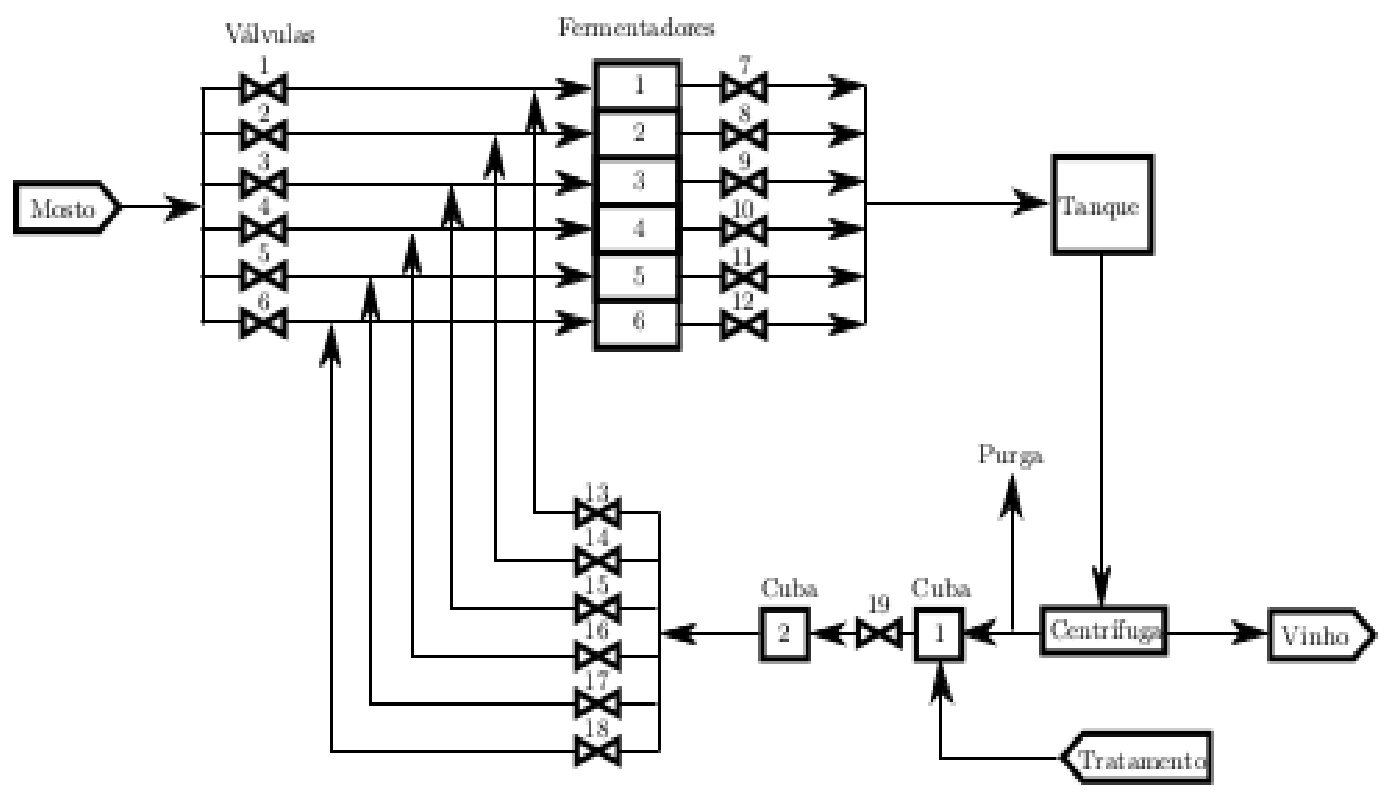

Figura 2 - Diagrama de blocos do processo de fermentação batelada alimentada.

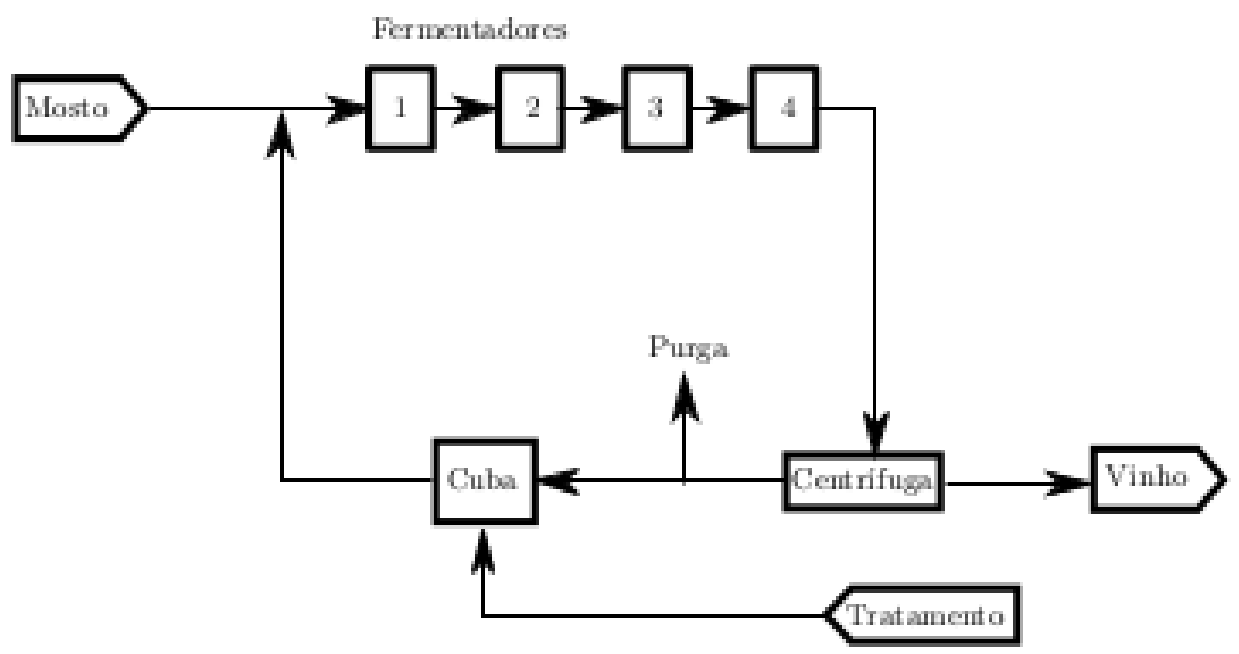

Figura 3 - Diagrama de blocos do processo de fermentação contínua.

A cinética de fermentação foi modelada obedecendo à Equação 1, proposta por Ghose e Tyagi (1979) e que considera os efeitos de inibição pelo produto e pelo substrato. As Equações 2 e 3 mostram respectivamente os balanços de massa global e por componentes. Na operação contínua e em regime estacionário, as derivadas temporais nas Equações 2 e 3 são nulas e as funções $F_{e}(t)$ e $F_{s}(t)$ são constantes. 

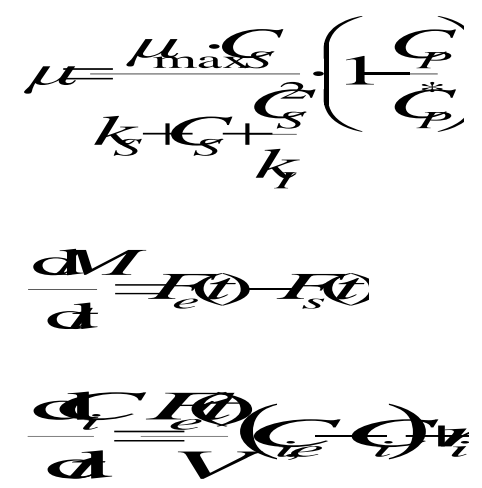

Considera-se que a sacarose converte-se muito rapidamente em glicose dentro dos fermentadores. As velocidades de reação da glicose, das células e do etanol são dadas pelas Equações 4 a 6 . A Tabela 1 mostra os valores dos parâmetros utilizados nos balanços de massa.

$$
\begin{aligned}
& r_{S}=-\frac{\mu \cdot C_{X}}{Y_{X / S}} \\
& r_{X}=\left(\mu t k_{D}\right) \cdot C_{X} \\
& r_{P}=\frac{\mu \cdot C_{X}}{Y_{X / P}}
\end{aligned}
$$

Tabela 1 - Parâmetros utilizados nos balanços de massa e energia.

\begin{tabular}{|c|c|}
\hline Parâmetro & Valor \\
\hline$\mu_{\max }$ & $0,36 \mathrm{~h}^{-1}$ \\
\hline$k_{S}$ & $0,48 \mathrm{~kg} / \mathrm{m}^{3}$ \\
\hline$k_{I}$ & $203,5 \mathrm{~kg} / \mathrm{m}^{3}$ \\
\hline$k_{D}$ & $0,0083 \mathrm{~h}^{-1}$ \\
\hline$C_{P}{ }^{*}$ & $88,0 \mathrm{~kg} / \mathrm{m}^{3}$ \\
\hline$Y_{X / S}$ & 0,024 \\
\hline$Y_{X / P}$ & 0,054 \\
\hline
\end{tabular}

\section{RESULTADOS E DISCUSSÃO}

Nas duas simulações considerou-se uma destilaria processando $500 \mathrm{t} / \mathrm{h}$ de cana com $13 \%$ de sacarose em massa, $71 \%$ de água e $16 \%$ em outros componentes. Nessas condições são produzidos cerca de $330 \mathrm{t} / \mathrm{h}$ de mosto contendo $18,7 \%$ em sacarose. As vazões das correntes de tratamento de creme de levedura, no entanto, foram consideradas diferentes nas simulações batelada alimentada $(115 \mathrm{t} / \mathrm{h})$ e contínua $(15 \mathrm{t} / \mathrm{h})$ e as produtividades dos sistemas de fermentadores não foram otimizadas, de forma que está fora do escopo deste trabalho comparar os desempenhos das duas configurações 
entre si.

A Figura 4 exibe o volume e os perfis de concentração de substrato, células e etanol em um fermentador batelada alimentada ao longo de um período de $12 \mathrm{~h}$. Cada período compreende um estágio de inoculação do creme de levedura com duração de uma hora, um estágio de enchimento com duração de quatro horas, um estágio de batelada de três horas, um estágio de descarga com duração de uma hora e um estágio de limpeza e inatividade de três horas. Os seis fermentadores operam defasados em duas horas um em relação ao outro. O vinho é armazenado em um tanque e posteriormente é centrifugado, produzindo 417,5 t/h de vinho delevedurado, que alimenta as colunas de destilação. São produzidas 360,2 t/h de vinhaça, 29,3 t/h de flegmaça e 27,9 t/h de etanol hidratado a 93,5\% em base mássica.

O reator simulado opera com produtividade muito baixa, de $4,40 \mathrm{~kg} / \mathrm{m}^{3} \cdot \mathrm{h}$, o que é evidenciado pelo substrato fornecido ser consumido rapidamente após o enchimento de mosto. Isso está relacionado ao tempo escolhido para a duração do ciclo de batelada alimentada ser longo demais para a quantidade de inóculo introduzido nos fermentadores. Outra dificuldade em relação à simulação do sistema batelada alimentada foi a necessidade de um tempo relativamente longo para a simulação ser concluída, em torno de vinte minutos. Isso torna o uso de modelos de conversão estequiométrica mais atraente para aplicações que envolvam muitas resoluções sucessivas do sistema, como otimização e análises de sensibilidade paramétrica.

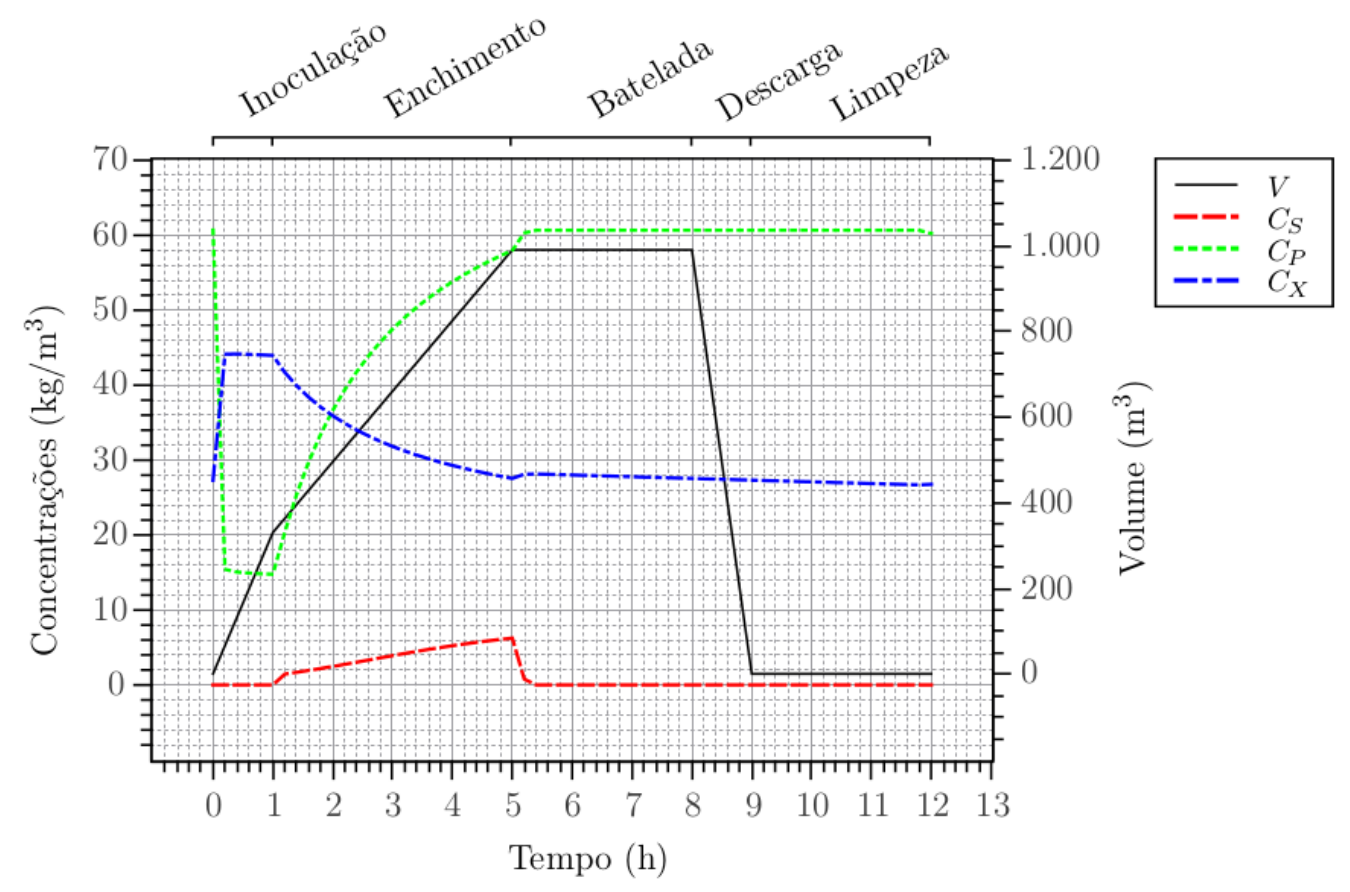

Figura 4 - Volume e concentrações de substrato, etanol e células em um fermentador batelada alimentada ao longo de um ciclo de fermentação. 
A Figura 5 mostra o perfil das frações mássicas de substrato, biomassa celular e etanol na corrente de mosto misturada à corrente de inóculo (posição 0 no eixo das abscissas) e nos fermentadores contínuos em estado estacionário (posições 1 a 4). A conversão obtida no sistema de fermentadores contínuos simulado está relativamente baixa, com valor próximo a $91 \%$ e a produtividade do sistema é de $7,70 \mathrm{~kg} / \mathrm{m}^{3} \cdot \mathrm{h}$. Nessas condições são produzidas $338,0 \mathrm{t} / \mathrm{h}$ de vinho delevedurado, 283,3 t/h de vinhaça, 24,6 t/h de flegmaça e 30,1 t/h de etanol hidratado com teor de 93,5\% em massa.

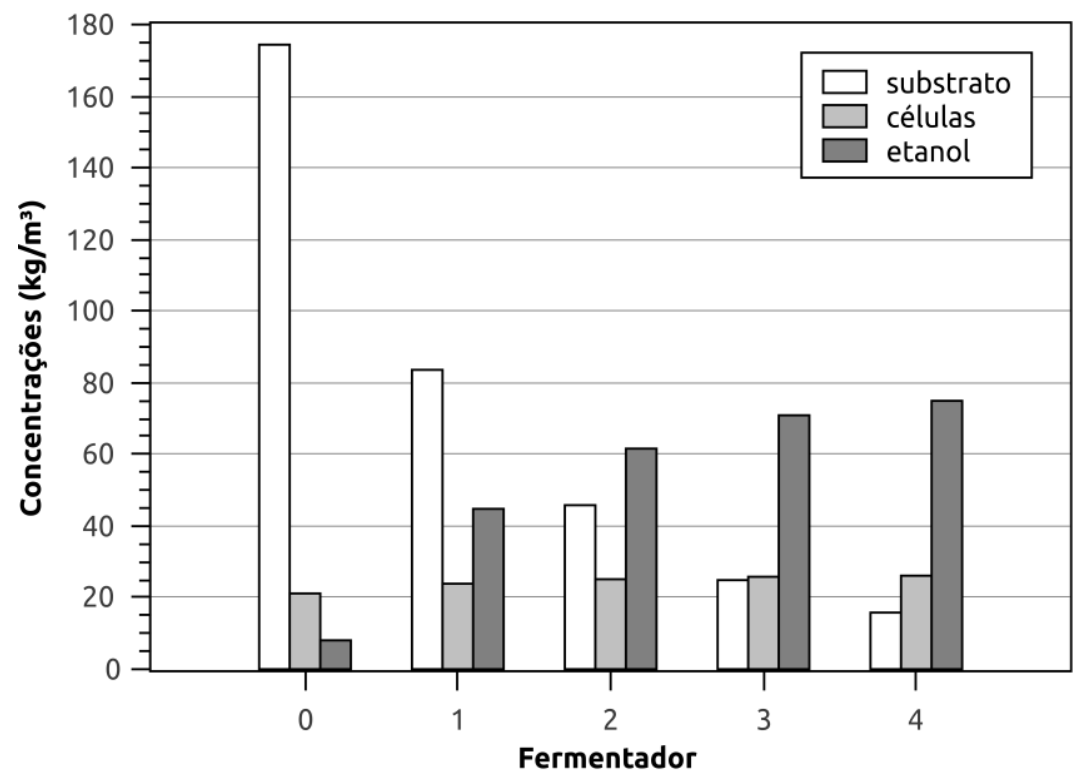

Figura 5 - Perfil das frações mássicas de substrato, células e etanol no mosto misturado ao fermento e nos fermentadores contínuos operando em estado estacionário.

\section{CONCLUSÕES}

O trabalho foi bem sucedido em produzir modelos para os principais equipamentos envolvidos no processo de fermentação alcoólica e construir e simular uma destilaria autônoma de etanol envolvendo as etapas de recepção da cana-de-açúcar, extração e preparo do caldo, concentração do mosto, fermentação e destilação. A destilaria foi simulada tomando como base de cálculo o processamento de 500 toneladas por hora de cana-de-açúcar com teor de sacarose de $13 \%$ em massa. $\mathrm{O}$ processo em batelada alimentada, implementado com um sistema de seis fermentadores em paralelo, produziu 27,9 t/h de etanol hidratado a 93,5\% em massa e 360,2 t/h de vinhaça. O processo contínuo estacionário, implementado com um sistema de quatro fermentadores em série, produziu $30,1 \mathrm{t} / \mathrm{h}$ de etanol hidratado a $93,5 \%$ em massa e $283,3 \mathrm{t} / \mathrm{h}$ de vinhaça. Nenhum dos processos, no entanto se encontra otimizado neste estágio do trabalho, tendo sido observada uma produtividade de $4,40 \mathrm{~kg} / \mathrm{m}^{3} \cdot \mathrm{h}$ para a operação batelada alimentada e $7,70 \mathrm{~kg} / \mathrm{m}^{3} \cdot \mathrm{h}$ para a operação contínua em regime estacionário. Futuros trabalhos podem avaliar diferentes condições operacionais com vistas a otimizar a operação da planta. 


\section{NOMENCLATURA}

C Concentração $\left(\mathrm{kg} / \mathrm{m}^{3}\right)$

$C_{p}{ }^{*}$ Concentração crítica de etanol $\left(\mathrm{kg} / \mathrm{m}^{3}\right)$

F Vazão $(\mathrm{kg} / \mathrm{h})$

$M \quad$ Massa $(\mathrm{kg})$

$k_{D} \quad$ Constante de morte celular $\left(\mathrm{h}^{-1}\right)$

$k_{I} \quad$ Constante de inibição pelo substrato $\left(\mathrm{kg} / \mathrm{m}^{3}\right)$

$k_{S} \quad$ Constante de meia velocidade $\left(\mathrm{kg} / \mathrm{m}^{3}\right)$ $r \quad$ Velocidade de reação $\left(\mathrm{kg} / \mathrm{m}^{3} \cdot \mathrm{h}\right)$

$t \quad$ Tempo (h)

$V \quad$ Volume $\left(\mathrm{m}^{3}\right)$

$Y_{X / P} \quad$ Coeficiente global de rendimento células/produto

$Y_{X / S} \quad$ Coeficiente global de rendimento células/substrato

$\mu \quad$ Velocidade específica de crescimento celular $\left(\mathrm{h}^{-1}\right)$

$\mu_{\max } \quad$ Constante de velocidade de crescimento celular $\left(\mathrm{h}^{-1}\right)$

\subsection{Lista de subscritos}

i Substância química qualquer

s Valor de saída

e Valor de entrada

S Substrato

P Etanol

X Biomassa celular

\section{AGRADECIMENTOS}

Os autores agradecem à CAPES e à FAPESP pelo apoio financeiro.

\section{REFERÊNCIAS}

CONAB (Companhia Nacional de Abastecimento). Acompanhamento da safra brasileira - cana-deaçúcar. 2013. <http://www.conab.gov.br/OlalaCMS/uploads/arquivos/13_04_09_10_30_34_boletim _cana_portugues_abril_2013_4o_lev.pdf>. [Online;acessado em 30 de setembro de 2013].

DIAS, M. O. S.; JUNQUEIRA, T. L.; JESUS, C. D. F.; ROSSELL, C. E. V.; MACIEL FILHO, R.; BONOMI, A. Improving second generation ethanol production through optimization of first generation production process from sugarcane. Energy, v. 43, n. 1, p. 246-252, 2012. 
EPE (Empresa de Pesquisas Energéticas). Balanço energético nacional - Resultados preliminares, ano base 2011. Ministério de Minas e Energia. 2012. <https://ben.epe.gov.br/downloads/Resultados _Pre_BEN_2012.pdf>. [Online; acessado em 15 de maio de 2013].

FURLAN, F. F.; COSTA, C. B. B.; FONSECA, G. C.; SOARES, R. P.; SECCHI, A. R.; CRUZ, A. J. G.; GIORDANO, R. C. Assessing the production of first and second generation bioethanol from sugarcane through the integration of global optimization and process detailed modeling. Computers \& Chemical Engineering, v. 43, p. 1 - 9, 2012.

GHOSE, T. K.; TYAGI, R. D. Rapid ethanol fermentation of cellulose hydrolysate. ii. product and substrate inhibition and optimization of fermentor design. Biotechnology and Bioengineering, Wiley Online Library, v. 21, n. 8, p. 1401-1420, 1979.

PALACIOS-BERECHE, R.; MOSQUEIRA-SALAZAR, K. J.; MODESTO, M.; ENSINAS, A. V.; NEBRA, S. A.; SERRA, L. M.; LOZANO, M. A. Exergetic analysis of the integrated first- and second-generation ethanol production from sugarcane. Energy, v. 62, n. 0, p. 46 - 61, 2013.

RFA (Renewable Fuel Association). Acceletaring industry innovation - 2012 ethanol industry outlook. 2012. <http://ethanolrfa.3cdn.net/d4ad995ffb7ae8fbfe_1vm62ypzd.pdf>. [Online; acessado em 1 de outubro de 2013].

SOARES, R. P., SECCHI, A. R. EMSO: A new environment for modelling, simulation and optimisation. Computer Aided Chemical Engineering. Volume 14, Issue C, 2003, Pages 947-952 DOI: 10.1016/S1570-7946(03)80239-0

UNICA (União da Indústria de Cana-de-Açúcar). Estimativa da safra 2013/2014.abril 2013. <http://www.unica.com.br/download.php?idSecao=17\&id=12655382/>.[Online; acessado em $15 \mathrm{de}$ maio de 2013]. 